\title{
Drowsiness Driving Prevention System using Bone Conduction Device
}

\author{
SangWoo Hahm ${ }^{1}$ and Hyungwoo Park ${ }^{2 *}$ \\ ${ }^{1}$ College of Business Administration, Soongsil University, \\ 369 Sangdo-Ro, Dongjak-Ku, Seoul, Republic of Korea (06978) \\ [e-mail: bload@ssu.ac.kr] \\ ${ }^{2}$ School of Electonic Engineering,, Soongsil university, \\ 369 Sangdo-Ro, Dongjak-Ku, Seoul, Republic of Korea (06978) \\ [e-mail: pphw@ssu.ac.kr] \\ *Corresponding author: Hyungwoo Park
}

Received February 28, 2019; revised May 30, 2019; revised July 27, 2019, accepted September 15, 2019; published September 30, 2019

\begin{abstract}
With the development of IT convergence technology, autonomous driving has gradually developed; however, the vehicle is still operated by the driver, who should always be in good health - but sometimes, this is not the case. It is especially dangerous to drive when drowsy, and unable to fully concentrate on driving, such as when taking certain medicines, or through fatigue. Drowsy driving is at least eight times more dangerous than normal driving, and as dangerous as drunk driving. Previous research has looked at technology to detect drowsiness, in order to wake up drivers when necessary, or to safely stop the vehicle. Furthermore, many studies have been conducted to find out when drowsiness occurs. However, it is more desirable for the driver to take sufficient rest during a break, in order to be able to continue to focus and drive. In other words, it is important to maintain a normal state before drowsiness. In this study, we introduce a sound source to increase driver concentration and prevent drowsiness, another that can improve the quality of sleep, and a system that produces these sound sources. The proposed system has a noise reduction effect of about $15 \mathrm{~dB}$. We have confirmed that the proposed sound induces an EEG of the desired form.
\end{abstract}

Keywords: Drowsiness Driving, Bone Conduction device, Electroencephalogram, Induction sound

A preliminary version of this paper was presented at ICONI 2018, and was selected as an outstanding paper. 


\section{Introduction}

Advances in technology have allowed people and goods to move about using the wheel. After the Industrial Revolution, because of the appearance of the internal combustion engine, the importance of having a driver in charge of the operation became greater. Moreover, people need rest, as is especially true for a driver in control or steering. The type of rest can be divided into physical and mental. Sleep is needed for physical and spiritual rest; it alleviates physical fatigue and relaxes the muscles. While sleeping for mental rest, the brain, which controls our mind, is also told to take a break [1]. In other words, sleep is necessary for drivers, and not having sufficient sleep becomes a problem. It is especially dangerous to fall asleep or be drowsy while driving. There is a high probability of accidents in abnormal conditions while driving. In particular, if people cannot handle normal machines because of drug fatigue or stress, a serious accident can result [2], as is true not only for the driving of vehicles, but also for accidents that occur in people's everyday behavior [1] [2]. Alcohol in the body, from drinking, interferes with normal behavior and diminishes judgment, thereby interfering with safe driving in complex situations [3]. Like drinking, stress from lack of sleep or fatigue interferes with safe driving [3] [4]. Such unsafe driving because of drinking and sleepiness can cause serious accidents, even leading to death [4] [5]. Various techniques have been introduced and applied to prevent this [4 - 10].

Reference [6] introduces a new technology that allows an electronic device to judge the current state of the driver and the vehicle, to make an emergency stop, to stay in a lane, and to stop or otherwise avoid a front-to-rear collision. In addition, studies have been carried out to monitor the health status of the driver and change the operation of the vehicle before applying safety techniques [7] [8]. These studies have attempted to detect drivers' drinking, stress, drowsiness, and health problems of the driver [8] [9]. The technology to stop a vehicle safely and report driver abnormality has also been studied [6]. In the future, technologies for the safe operation of all vehicles will exist. Although the steps are not completed yet, it is not too far in the future that vehicles directly operated by people will likely disappear completely [10].

All of the above mentioned technologies to help safe driving operate when the driver cannot safely drive. If a vehicle can be alerted to situations in which the driver may be about to fall asleep, or even just have drooping eyelids or a heart rate different from usual, the vehicle may be able to stop in a safety zone by itself [6] [10]. But the important thing is that the driver must be aware of the situation and try to maintain consciousness. In other words, it is safe to stop the vehicle in a rest area. When a driver arrives at the rest area, he or she needs to sleep for a short time. But even if drivers do stop at a safe place, it is difficult to get sufficient rest because of light, noise, or discomfort of the seat.

The Korean highway system has regular resting places at average distances of $25 \mathrm{~km}$; when these are not present, there are small resting places every $15 \mathrm{~km}$ for drowsy drivers to park [11]. But problems exist for areas where there are no resting places or drowsiness shelters. In other words, if drowsiness occurs during certain 5-km intervals, a dangerous operation must be maintained for more than five minutes, and the probability of an accident happening during that time is eight times higher than it is normally [12]. The best thing for a person who needs a rest is to sleep. If we divide the types of mental state into sleep, sleepiness, nap, etc., each type of fatigue can be resolved, and normal thinking and behavior can resume after rest [2]. Normal sleep relieves physical and mental fatigue [1] [2]. For driving while drowsy, 
fatigue is not solved well, and if one continues to drive, normal behavior is lost and judgment becomes blurred [2].

Sound has various roles in human life. Certain sounds are related to human emotions (e.g., joy or sadness), including dangerous alarms and communication (voices). Sound also affects human brain waves in a variety of ways. Human auditory organs cannot be blocked by the voluntary muscles and constantly transmit sound signal information to the human brain. Furthermore, the auditory nerve is near the limbic cortex and so can stimulate associations with the brain and hearing. By this reaction, certain sounds can stimulate the brain and make people more focused. When sound affects the human brain, the EEG response can change. With other sounds, humans may be able to rest more effectively. Thus, herein we explore the relevance of the effects of certain sounds on human brain waves. When an individual hears a certain sound, the brain waves will change. When we identify the effect of a specific sound on brain waves, we can stimulate the human brain by means of sound. So, using the sound, individuals will be able to concentrate or relax more efficiently when they need to. These influences will help develop human potential, including student academic performance and work performance, and enable people to demonstrate their abilities. To illustrate this process, we explain the relevance of individual electroencephalography (EEG) analysis to cognition when listening to specific sounds [13].

Human hearing can react very sensitively [14]. Using this characteristic, the system should not allow sleep from the moment of detecting sleepiness; rather, it will play a sound that induces a mental state that allows the driver to stay focused on driving. However, if drivers set the mode to allow them to rest, the system will reduce the noise around them and induce a good night's sleep. In previous studies, sound has been used to induce certain types of EEG [15-18]. In this study, we exploit the properties of these sounds to induce mental and physical awakening or resting states in drivers. We insert an independent sound system in the vehicle audio system in the seat headrest and in the driver's seat of a vehicle; this system reproduces sounds that can induce the mental state corresponding to the arousal necessary for driving, as in the studies of Refs. [17] and [19]. During a rest break, we introduce a systematic method of producing a resting sound that allows a good night's sleep and that can also allow high-quality rest for short periods.

\section{Related Works and Research Background}

\subsection{Danger of drowsy driving}

Sleep is an unconscious state in which an animal rests for a certain time in body and mind. Consciousness is diminished, and sensory organs are relatively inactive and characterized by almost no movement of the voluntary muscles [2]. Sleep is distinguished from arousal by a decreasing response to stimulation and is distinguished from hibernation or coma [2]. During sleep, assimilation increases and the immune, nerve, bone, and muscular systems experience recovery [2]. Sleep is found in all birds and mammals, and in many reptiles, fish, and amphibians. The purpose and mechanisms of sleep have been only partially understood, and active research is under way [2]. Generally, adults spend about five to eight hours a day sleeping. People deliberately adjust their sleep times. The average sleep time for people over 15 years old in Korea is about 6 hours and 15 minutes, and in the United States is about 7 hours [20]. If people do not have enough sleep, they will feel fatigued and their emotions will become sharp, which makes it easy for them to get irritated or angry. In addition, if sleep deprivation persists for a long time, the likelihood of suffering from various diseases, 
such as cardiovascular disease or mental illness, increases [20]. In other words, lack of sleep leads to side effects of loss of bodily or mental control. Dangers in the surroundings in these circumstances can present themselves. In particular, if people engage in drowsy driving, they will be threatened, as well as their surroundings.

Drowsiness is a condition close to sleep, an intense desire to sleep; it is a condition that does not usually persist for long and usually indicates a state before sleep [21]. It may be accompanied by lethargy, weakness, and a lack of agility [21]. Drowsiness is often considered a symptom, not a disability in itself. However, for certain reasons, drowsiness repeated at specific times can present as a variety of disorders, including excessive daytime sleepiness and nighttime sleep disturbance [21]. Sleep deprivation sometimes causes drowsiness; in this case, people are not able to control the body well, the mind often becomes careless, and accidents often occur. If the brain is not fully awake, it cannot engage properly with the world and makes mistakes, including being unable to move [21]. Social problems caused by lack of sleep and drowsiness of people driving cars are very damaging. Especially bus drivers and sleeping drivers of large trucks can cause large and damaging accidents. Drowsy driving causes the highest rate of accidents in Korea. This rate is higher than the accident rate associated with alcohol [3] [4]. Factors that interfere with safe driving include medications such as alcohol, and states such as drowsy driving. Unhealthy mental and physical states can cause driving carelessness and deterioration of concentration [5]. By means of examples and regulations, we identify the dangers of drowsy driving [5].

\subsection{Safety Assistive Technology (Drowsy Driving Prevention)}

Safety Assistance Technology, introduced by Refs. [4], [6] and [10], allows a vehicle to operate itself by artificial intelligence, to diagnose the status of the driver and the vehicle, and to take necessary measures. These technologies can be basically divided into sensing techniques for judging whether a driver is performing normally and techniques for operating the vehicle [4] [10]. Studies have been developed to identify a driver's vital signs and to measur the driving conditions of their vehicles. In order to diagnose a driver's condition, images, sounds, electrocardiograms, blood oxygen saturation, and EEGs have been used [4] [6]. In recent years, by means of machine learning, such as artificial intelligence and especially deep learning, research has been carried out to identify a driver's biological condition using limited sensors [4]. The state of a vehicle and driver is evaluated first; the control system of the vehicle also accumulates data for safe operation by means of machine learning. Figure 1 summarizes these techniques. This system is the part that largely detects the condition of the vehicle, the condition of the driver, and the operating environment. This system is monitored and controlled by artificial intelligence. It generates data on driver alertness, vehicle control, and emergency rescue signals. Briefly, there are three stages of detection, judgment, and control [6]. Unless the system is at a fully autonomous level of SAE 5, as in Ref. [10], the driver must be able to operate the vehicle normally and be prepared for emergencies. That is, functions to date can intervene when drowsiness occurs, but it is important to relieve fatigue so that drowsiness does not occur.

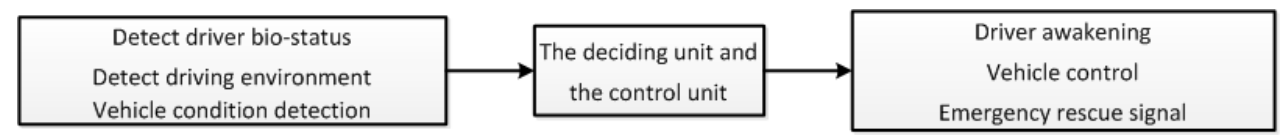

Fig. 1. Active Noise Reduction Method Using Cancellation Interference 
When a person drives, accidents can happen, such as when a vehicle is not controlled because of a fault in the vehicle, driver carelessness, a natural disaster, or the natural environment. A variety of people and goods such as passenger cars, buses, and trucks ride on roads. As such, unexpected accidents can cause great damage. In particular, accidents caused by drowsy driving can be very serious and can lead to death. Therefore, in order to prevent such accidents, various studies are being conducted to confirm the health and condition of drivers. These studies basically judge a driver's bio signals, corresponding to the first step in Fig. 1, by means of a contactless sensor. The sensor also gauges fatigue using the degree of a driver's head droop (angle, distance) [22-25]. Brain waves are analyzed by means of simulation experiments [8] and learned signals are found by means of machine learning, such as by using a neural network [26]. Another study was conducted using a water cluster, a noncontact sensor, to simultaneously detect drowsiness and drinking by using alcohol measurement and breathing cycles [27]. A method of sensing changes of driving posture using a pressure sensor in the driver's seat was also proposed [28]. In addition, the front camera of a smartphone was used to judge driver eyelids and give warnings [29]. There was also a study to find out the possible degree of involvement of artificial intelligence systems in vehicle control [30]. In Ref, [31], the driver's heart rate was sensed. In addition, research [31] [32] to measure sleepiness by means of machine learning analysis of electrocardiogram (ECG) was conducted, as was a study to find out what degree of intervention could be accomplished by the operation of fuzzy logic [33].

Studies that contribute to safe driving will use contact electrode sensors (for the EEG) and non-contact (camera) sensors to monitor an actual driver's waking state. There have been a few studies on devices or techniques that can keep drivers from falling asleep. In the not too distant future, there will be completely autonomous vehicles on the road. But until then, individuals have to control vehicles. Driving safely and improving the efficiency of short breaks are basic conditions for everyone's safety. As we saw in section 2.1, a short rest, with low- quality sleep, causes chronic fatigue, which causes drowsy driving. High-quality relaxation and concentration on driving induced by the stimulation of human auditory organs and brain waves are necessary.

\subsection{Human Auditory Characteristic and electroencephalogram}

The ear is the organ responsible for listening, It can be anatomically divided into the internal ear, middle ear, and external ear. The ear corresponds to a transducer that, using a little energy, converts sound into an electric signal that travels by means of the auditory nerve [13]. "The human ear evaluates external acoustic signals, and is the basic sensory organ that grasps and judges the types and characteristics of sounds that occur in a person's surroundings and transmits them to the brain [34:p. 59].” These elements not only evaluate the sound based on subjective criteria, but also provide a basis for understanding information by means of the objective sound obtained by these senses. The process by which humans perceive sound is one in which the sound source is transmitted by means of the air and then transmitted as an electrical signal in the inner ear's cochlea, after which it is recognized by the brain [35]. Unlike human vision, auditory signals are difficult to intercept, and constantly transmit information to the brain. Sound endlessly affects the brain, causing changes in brain waves or in psychology. In addition, objective and subjective evaluation of sounds can produce a good indicator of sound [13] [34]. 
Electroencephalography (EEG) is an electronic physiological monitoring result that records the activity of the brain. EEG is typically used to distinguish several waves, such as Alpha, Beta, Delta, Theta, and Gamma waves. It is usually non-invasive and the electrodes are placed along the scalp. Invasive electrodes are also used for certain applications. EEG means that the voltage change of neurons due to brain activity is measured on the epidermis. Diagnostic applications typically focus on the spectral content of brain waves, which is the type of neural vibration that can be observed in brain wave signals (commonly called "brain waves") [37].

The types of EEG can be divided into slow wave and fast wave. Their characteristics can be classified according to the analyzed frequency [36] [37]. Table 1 describes the types and features of brain waves recorded by an EEG. One of the waves separated from Table 1 does not appear as $100 \%$, but the feature and state are divided according to the wave of the main component in a specific state. In general, the alpha wave, which is known to be good for studying or concentrating, seems to have a lot of actual meditation and relaxed EEG states. However, maintaining tension in the midst of the alpha and beta waves helps to maintain a concentrated state [15] [17]. Previous studies have shown that EEG is induced by means of sound [15 - 18] [36] [37]. In addition, as mentioned earlier in this section, human hearing organs cannot be blocked by the voluntary muscles, are directly connected to the brain by means of the hearing cells and nerves from the inner ear, and have an influence and induction of the human condition and brain condition. Reference [18] analyzed the characteristics and psychological state of the I-Doser sound, called cyber drugs. The background research confirms that a specific sound helps to control the EEG and human state [15 - 19] [36 - 38]. Previous studies have shown that the EEG is changed and induced by specific sounds. The auditory nerve is near the limbic cortex, so can stimulate associations with the brain and hearing. By this reaction, certain sounds can stimulate the brain and make people more focused [15 - 19] [36] [37].

Human beings perceive specific objects. This cognition affects human attitudes. Furthermore, attitudes are closely related to human behavior [38] [39]. Thus, when an individual perceives a specific sound, his or her attitude may change as well as the EEG, so he or she will be affected by specific sounds in terms of their attitudes and behaviors (e.g., concentration or relaxation) by specific sounds. For example, if a person is listening to fast tempo music, a change in the EEG occurs in the fast wave. Sometimes people feel less tired when they work with marching songs or work songs. Also, people listen to white noise, which is the natural sound for meditation. An artificial and very powerful sound like i_Doser also makes the state of EEG by a specific drug .

Table 1. Types and Features of Brain Waves recorded by an EEG [15]

\begin{tabular}{|c|c|c|}
\hline Class & $\begin{array}{c}\text { Indicator, Frequency } \\
\text { Definition }\end{array}$ & Features and State \\
\hline \hline \multirow{3}{*}{ Slow wave } & Delta wave(0.1-3Hz) & When people fall into deep sleep. \\
\cline { 2 - 3 } & Theta wave(4-7Hz) & $\begin{array}{c}\text { Twilight sleep between deep sleep and weak } \\
\text { wakefulness. }\end{array}$ \\
\cline { 2 - 3 } Fast wave & Alpha wave(8-12Hz) & $\begin{array}{c}\text { When a person is in a relaxed, passive, and un- } \\
\text { concentrated state. Meditation }\end{array}$ \\
\cline { 2 - 4 } & Beta wave(13-30Hz) & $\begin{array}{c}\text { When a person is dealing with specific and specific } \\
\text { problems. Tension, Excitement, Stress. }\end{array}$ \\
\cline { 2 - 3 } & Gamma wave(30-50Hz) & $\begin{array}{c}\text { When people is nervous condition, reasoning, an } \\
\text { irritated state or mystery. Anxiety, Nervous, Stress. }\end{array}$ \\
\hline
\end{tabular}




\subsection{Active Noise Cancelation}

Sound is generated by phenomena such as friction, collision, bouncing, air flow, and explosions. In the process of generating such sound, kinetic energy, position energy, or chemical energy is converted into another energy form, causing the surrounding air molecules to vibrate. The fundamental principle is that the vibrating waves of the air molecules push and pull the surrounding air molecules to form the wavefront of the sound and spread out. The vibrations of the generated longitudinal waves are conveyed by the medium, and the physical phenomenon, heard in the ear as sound, can be analyzed in terms of the generation method, the resonance obtained in the transmission process, and the characteristics of the filtering. Two or more sounds influence each other in the course of sound transmission. The three elements of sound analysis are volume, frequency, and duration. These three elements are independent of each other and can be distinguished and analyzed, making it useful to change or create a sound to fit a situation [14] [35] [40].

Since the sound propagates by means of the atmosphere, once it is made, it will naturally affect all the surrounding areas until it diffuses and disappears. Especially in the auditory organs, small changes can be sensitively received. "Noise" means all the sounds that the listener does not want to hear. One way to reduce the influence of such noise is to use active noise-reduction methods that involve masking or reducing using opposite-phase sounds. Sound is tremors of the air, spreading around. In the process of spreading, two or more sounds cause mutual interference, which is characteristic of wave motion. If the phases are opposite to each other during the mutual interference, the air at that point is not disturbed; this principle is used to reduce noise. The principle is simple, but it is difficult to control sound in real 3D space. It is therefore important to ensure that no more than one system interacts with the space in close proximity to the ear [14] [35] [40]. The following equation describes the sound heard by people using a noise-reduction method.

$\mathrm{D}(\mathrm{t})=\mathrm{A}(\mathrm{t})+\mathrm{C}(\mathrm{B}(\mathrm{t}))$

Sound heard by a person : $\mathrm{D}(\mathrm{t})$

System output : A (t)

Phase processing : $\mathrm{C}$

Ambient noise : B (t)

In this case, ambient noise B (t) is phase, which is multiplied by $\mathrm{C}$, and the anti - phase noise of the ambient noise is reduced, so that the sound combined with the system output is reduced. Sound is constantly propagated to the surroundings by means of the air. At this time, if phase adjustment $\mathrm{C}$ is not correct, the system output $\mathrm{A}$ and the ambient noise $\mathrm{B}$ can be in phase with each other, and the noise will be amplified. Figure 2 illustrates the principle of noise reduction. If we perform phase processing C such that A and B in Eq. (1) are opposite in phase, the sound D (noise amplitude) heard by humans is reduced [16] [34]. In this study, rather than attempt to control distant sounds, bone-conduction speakers were used to control noise in the local space, and the bone-conduction amplifier was set to equal the boneconduction level [41] [42]. 


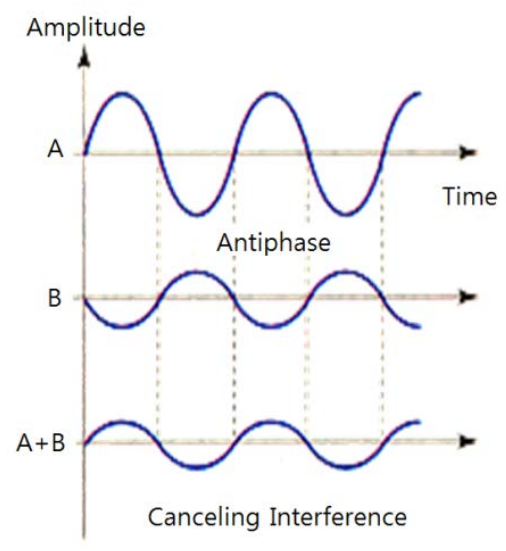

Fig. 2. Active Noise Reduction Method Using Cancellation Interference [14:p24, edited]

\section{Proposed method of drowsy driving prevention}

\subsection{Proposed system}

The proposed method reduces the noise of the vehicle to the driver of the vehicle, especially the functional sound to the driver while driving. That sound consists of natural sounds and keeps concentrating sound. Also, in a resting area, the mode can be switched to provide an efficient resting sound for a short time. In previous studies, we investigated how the human condition was induced by the use of sound and music [16] [17]. We confirmed that the sound that maintained concentration was a natural sound, such as a white sound and a binaural sound inducing an alpha wave. The characteristic of this sound component is $11-13 \mathrm{~Hz}$, which is the high band of the alpha wave. By the way, the human auditory organ cannot recognize sounds below $20 \mathrm{~Hz}$. Therefore, in binaural sound, by adjusting the frequency difference between the sounds heard by the left and right ears, the frequency difference is recognized [40] [43]. The volume of the sound is about $6 \mathrm{~dB}$ larger than the ambient noise, so that it does not give the subject any sense of heterogeneity. As well, let it be mixed with ambient noise [14] [34]. The proposed acoustic system and the car audio system are configured independently. The existing car audio enables the radio channel or music to be played by the driver. The proposed functional sound is played separately from the car audio. The ANC system is configured near the headrest to reduce noise, such as engine noise or road surface friction noise generated during vehicle operation. Then, the proposed sound is efficiently supplied to the driver. A sound source that changes rapidly depending on the situation, such as the start of the system, the driver's seating, and the start of operation, can surprise the driver and cause a sense of discomfort. Therefore, the proposed method adaptively adjusts the size of the sound source so that it is increased by about $6 \mathrm{~dB}$ compared to the ambient noise [41 - 43].

Fig. 3 shows a model for the implementation of the proposed system. The headrest is equipped with a microphone noise monitor, a bone-conduction speaker, a general speaker, and the control unit. Because of the destructive interference described in Section 2.4, the controller outputs an anti-phase signal from the microphone to the bone-conduction speaker. Then, for each situation, the control unit supplies sounds to either improve concentration or to induce sleep in the driver. 


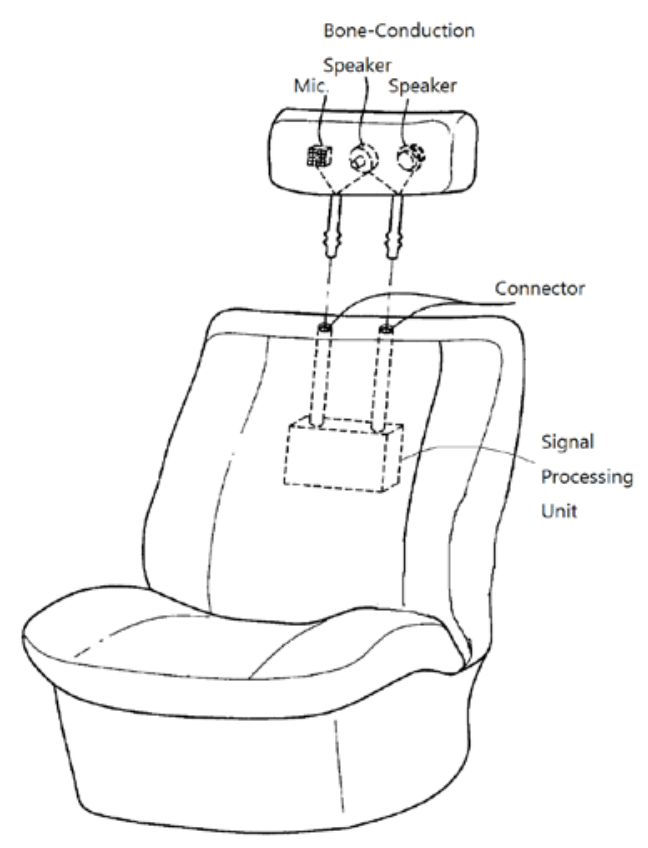

Fig. 3. Model diagram of the proposed driver seat [43:p9, edited]

Fig. 4 shows a block diagram of the signal flow for the model proposed in Figure 3. The driver can hear the noise from his or her vehicle and from the outside. When the driver operates the audio device of the vehicle, he or she will hear a mixture of three sounds. Noise is sounds that people do not want to hear. For the driver, the vibration and sound of the vehicle, which cause stress and fatigue, and the external sounds, are noise. Sounds corresponding to noise are canceled by adjusting the phase to reduce their influence. By means of the proposed system, in the vicinity of the headrest, the sound that is amplified is only the necessary sound with a little noise. Inside the system, there is a part that synthesizes phase adjustment and the prepared sound necessary for the driver. Depending on how much noise is received by the microphone mounted on the headrest, the system makes sure that the sound is of the proper volume; this adjustment includes the ability to adjust the volume of the sound produced by the speaker. Since the internal acoustic environment of a vehicle varies greatly both in size and frequency with time, it is necessary to adjust the sound volume according to the situation [34].

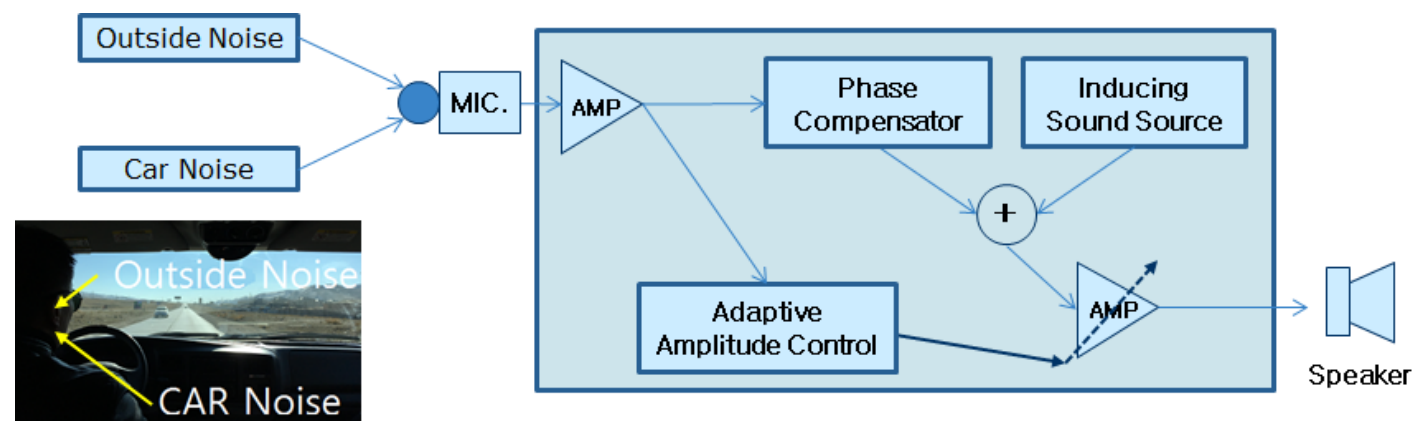

Fig. 4. Signal Flows of the proposed System 
The Inducing Sound Source is a sound that focuses on the type of driving that drivers need and that can also induce a good night's sleep. As studied in Refs. [15] [17] and [18] show that the effect of sound on the human brain is significant. In particular, because of the general characteristic of human hearing, it is not possible to close the ears in the way in which the eyes close. This characteristic is useful for keeping the brain in a state of wakefulness and concentration in driving and in various other situations. During driving, the device continuously reproduces sounds of nature and sounds that induce a high EEG state so as not to allow a driver to transit to a too-slow EEG. When stopping driving and during rest, the device relaxes tension and generates slower EEG-inducing sound. However, human hearing cannot be heard directly, even if it is the sound of a fast brain wave. Thus, Ref. [18] uses a binaural frequency sound to measure the difference of a single-frequency sound transmitted to the left and right ears. The method is less effective when listening by means of the air, and the person who listens to a single frequency becomes tired and bored easily. However, using multi-tones does not induce the proper EEG. The sound used in this study was synthesized by combining a binaural beat and natural sounds. Natural sounds are distributed evenly over a wide frequency band. The effect of the sound is as positive as in Refs. [15] and [17]. However, to maximize the driver's concentration and make it possible for a driver to enjoy a good night's sleep during a break, a 3-15 Hz sound, which cannot be heard directly with the synthesized binaural beat, is induced.

\subsection{Performance Test of the Acoustic System}

We tested the active noise-canceling performance and acoustic performance of the proposed device. For the experiment, we used Micro-Electro Mechanical Systems (MEMS) microphones and phase control IC, as below, and used an osteoconductive amplifier and bone-conduction speaker to construct a closed loop from mike to bone-micconduction output. Fig.s 5 and 6 show the devices used in the actual configuration. On the right side of Fig. 5, there is a MEMS Mike. An actual MEMS Mike is several millimeters in size and mounted on the expansion board. In Fig. 5, there is a phase-adjustment board in the center of the left side. The phase-control IC is composed of two terminals, the lower part of the figure, and the Mike, so that the input and the drowsy sound source can be mixed and output. The Mike and the output of the input match well and the output converges to zero. The results are shown in Fig 7. It is confirmed that the input and output signals operate in opposite phases for various frequencies and general noise. The graph shows the oscilloscope screen of time response.

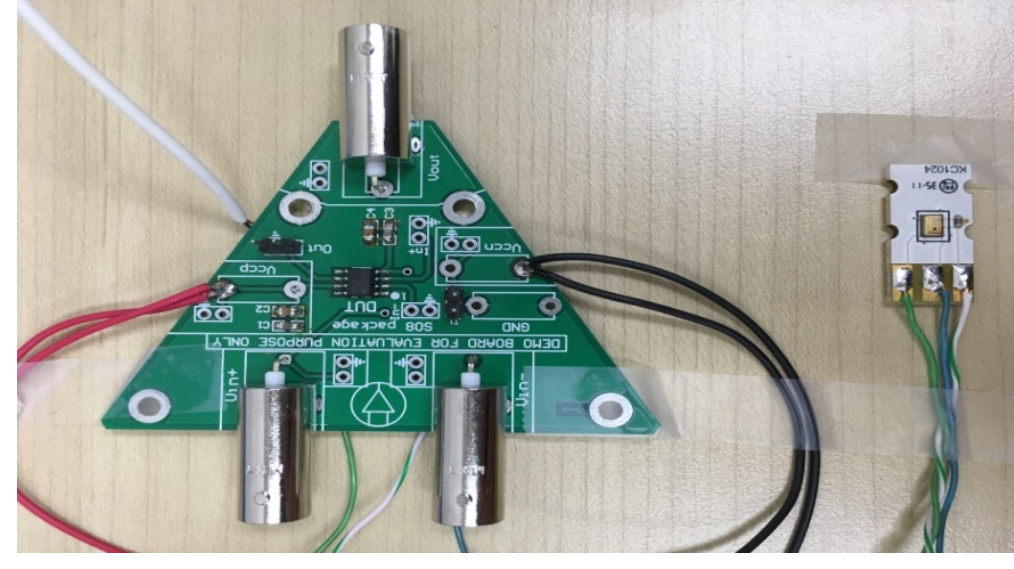

Fig. 5. Phase control board and MEMS Mic. 
The device is operated in such a way that the ANC of the device is divided into an open loop and a closed loop before and after the operation. Fig. 8 shows the frequency response for both situations. Overall, we can find that the sound near the speaker has been reduced, especially in the $1 \mathrm{k} \mathrm{Hz}$ and $4 \mathrm{k} \mathrm{Hz}$ bands, to which human hearing is sensitive. Using the difference between the two values, it is shown in Fig. 9 that the operation is not smooth at frequencies below $300 \mathrm{~Hz}$, but the overall response gain shows a positive value and works well. The overall average value is about $7 \mathrm{~dB}$, which is not the best performance of ANC, but the noise near the headrest is lowered by $7 \mathrm{~dB}$ and the noise reduction effect is increased by means of the intensive sound source or the resting sound source required for the driver.

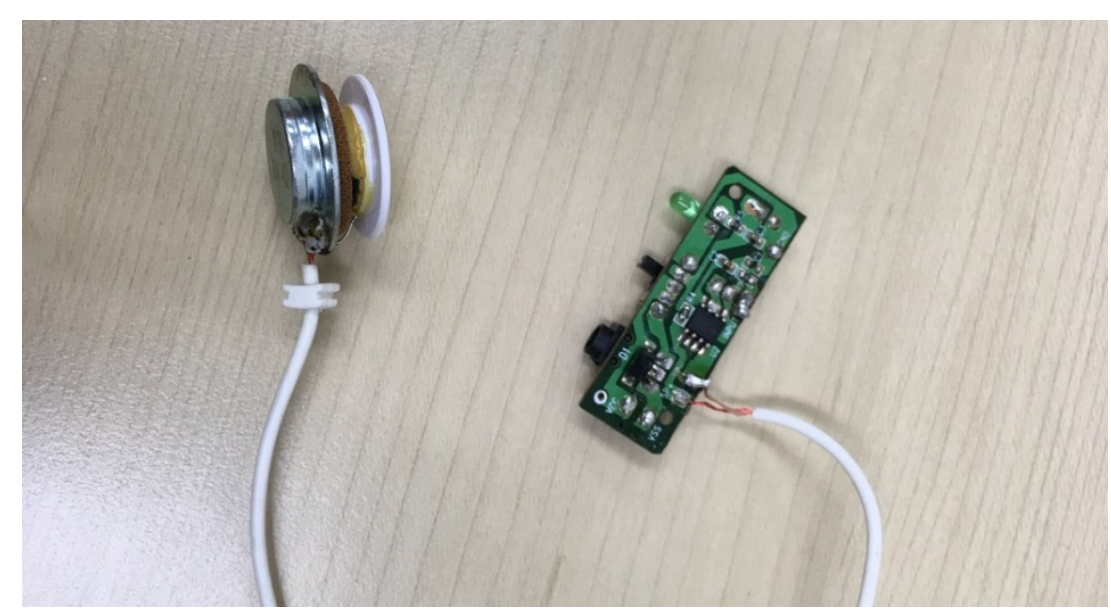

Fig. 6. Bone-conduction speaker and amp. board
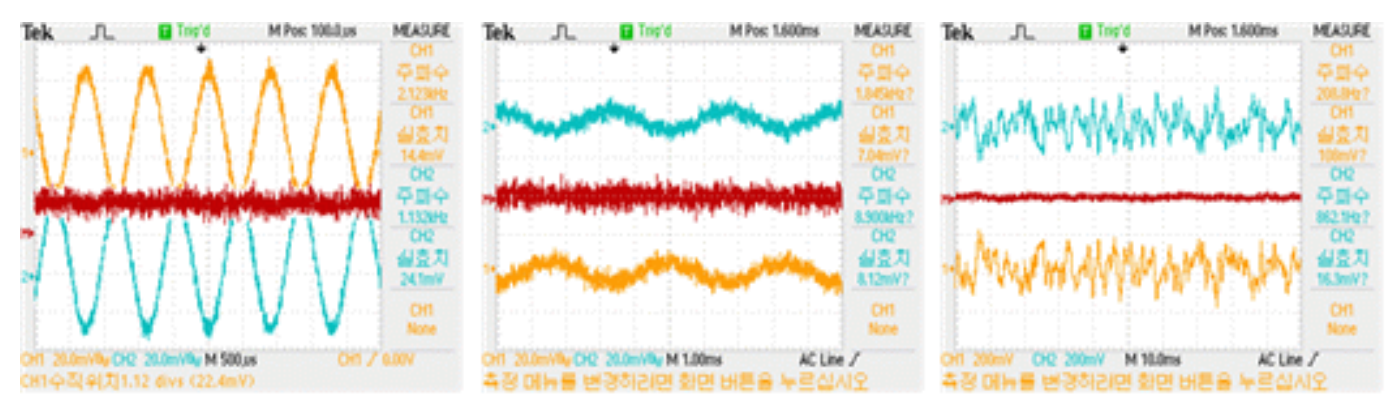

Fig. 7. Time-domain response 


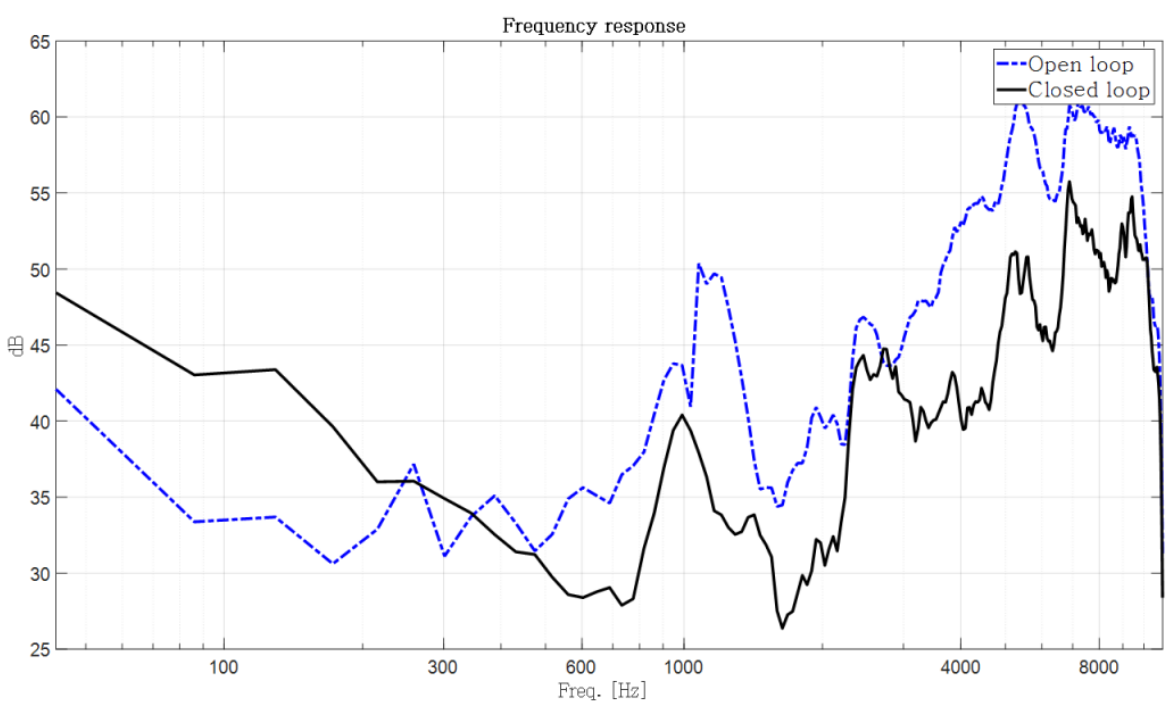

Fig. 8. Frequency response of open and closed loops

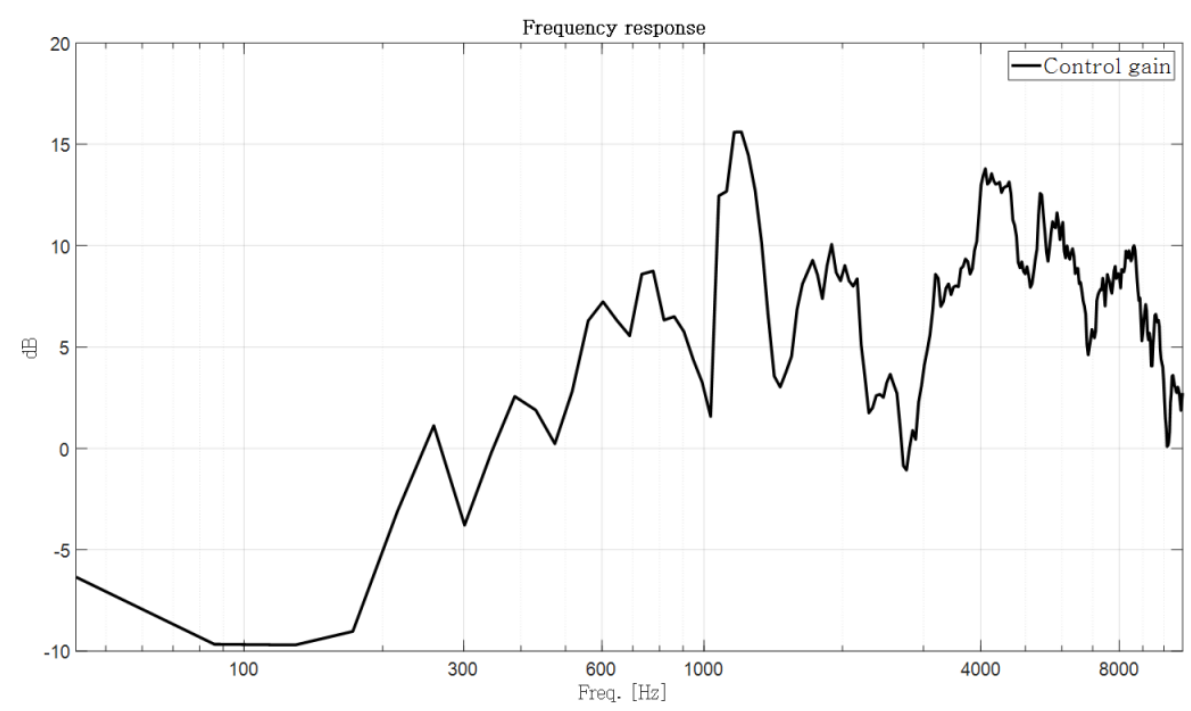

Fig. 9. Control gain of closed loop

\subsection{Proposed Drowsy driving Prevention Sound}

This study looks at a method to supply sounds that will prevent sleepy driving. ANC is performed in the headrest area using the bone-conduction speaker system. In a quiet environment, the sounds of nature, such as sounds of waves, and synthetic sounds using binaural beats keep the driver focused and prevent drowsiness. While taking a break, the proposed method reduces the ambient noise and reproduces sound that can induce a good night's sleep, so that driver can rest efficiently in a short time. Figure 10 shows an example 
of synthesized sounds that allow a driver to focus on driving. It shows the synthesized section that combines the sound of waves and the sound that induces concentration. We confirmed that the sounds of nature and the sound of the binaural beat are synthesized.

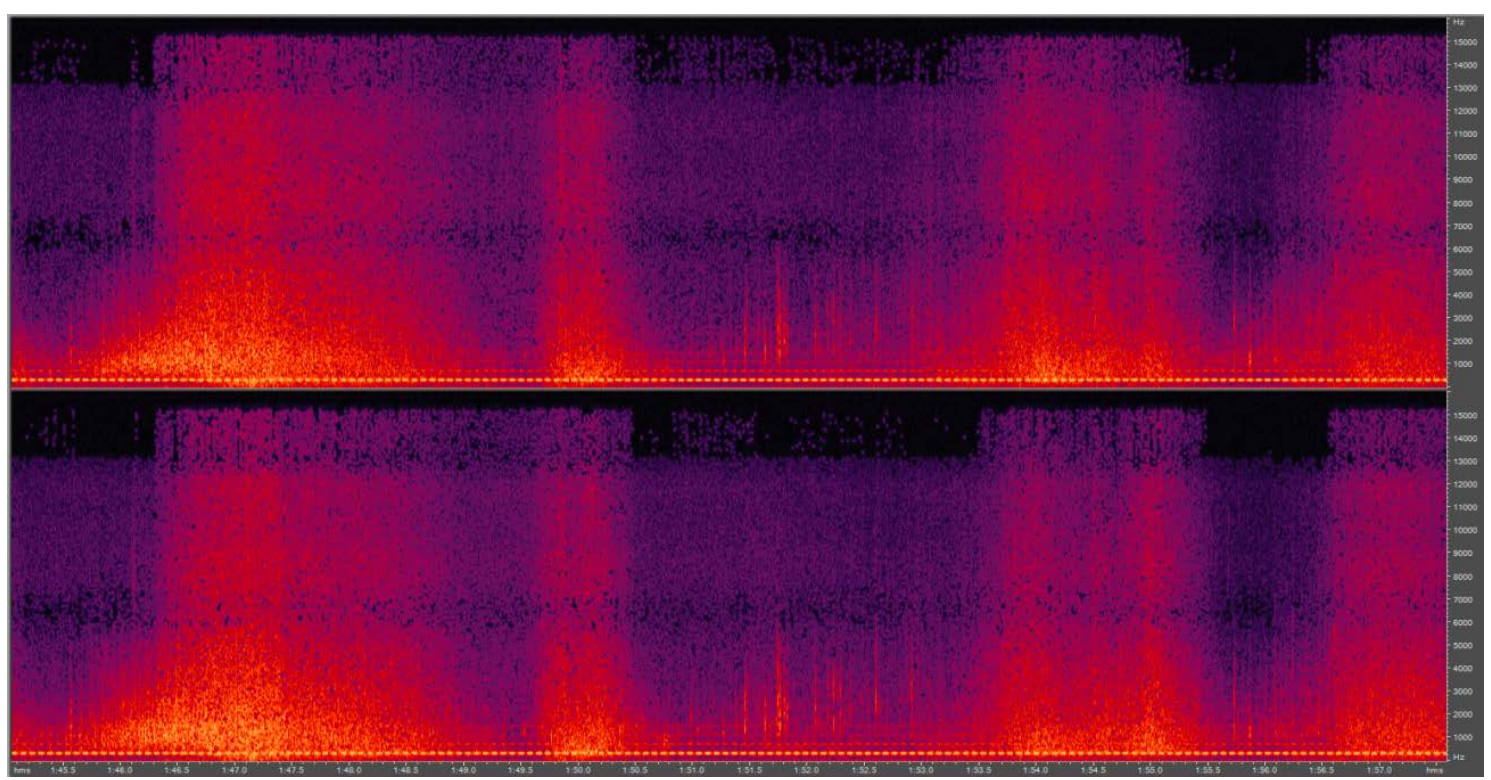

Fig. 10. Spectrogram analysis of the synthesis sound

It is a good idea to reduce the overall sound volume while taking a break. Even if a sound induces sleep, one cannot sleep when the sound is loud. The volumes of the bone-conduction ANC and the sleep-induction sound are sufficient to mask 10-15 dB of noise around the vehicle. Since the bone-conduction ANC device has a noise-blocking effect of $7 \mathrm{~dB}$, it is optimal to have a value of 3-8 dB compared to the ambient noise. Then, the proposed sound is actively adjusted according to the volume of the ambient noise, so that the average sound is masked by $15 \mathrm{~dB}$. Therefore, even if a driver hears these sounds for a long time (more than one hour), the ears and the brain will not be fatigued by the sound.

\section{Experimental Results}

\subsection{Experimental Environment}

It is dangerous in terms of safety to use the proposed system for direct driving and to check its effect. In order to construct an environment similar to that of actual operation, a black-box image recorded during operation was reproduced, and the noise generated at this time was reproduced in the vicinity by the monitor speaker at the measured volume. The concentrations of regenerated and non-regenerated regions of the sleeping sounds were analyzed by means of EEG analysis. From the beginning, we compared cases in which sleepiness-prevention sounds were reproduced together with sounds that did not induce sleepiness. During the experiment, the brightness of the test site was controlled. The subjects were male college students; they were asked to operate the system continuously for a total of two hours with a short rest time of one hour without the sleepiness-prevention sound, and 
one hour without sleepiness. After a two-hour measurement period, we observed the results after taking a ten-minute break while listening to a sound that induces a good night's sleep.

In the experiment, we used the two-channel day electrode named 'Neuro-Harmonies'. The sampling rate is $256 \mathrm{~Hz}$ and the measurement bandwidth is $0-45 \mathrm{~Hz}$. The communication with the PC is the USB type, and the CMRR is $0.6 \mathrm{v}$ VCM $<3.7 \mathrm{v}$. The product is a KCcertified MSIP-REM-BP1-NEUROHARMONYS. In the simulated operation, we made it possible to simply measure the signal from the forehead. It was difficult to use an EEG analysis system with a plurality of electrodes during the experiment. Neuro-Spec was used for the analysis software; its is verified by the manufacturer so that it can work well with the HW used in the measurement. Compared to the initial version, the stability and the analysis speed were improved, and the brain wave of the measurement can be stored and reanalyzed.

The experimental results show that the proper EEG was obtained, as shown in Figs. 11 and 12 The resulting graph was saved and attached to the screen of the EEG-analysis program. The figure is a spectrogram analysis of the measured EEG. Figure 11 shows the results of the measurement at the beginning of the experiment, while figure 12 shows those in the middle of the test. It can be seen that the various EEG responses are composed of different types of responses. Both figures display two kinds of data by three-dimensional time difference. The horizontal axis of the figure shows the flow of time, while the vertical axis shows the analyzed frequency value. The intensity of the EEG corresponds to the frequency in terms of color and height. Figures 11 and 12 show whether the brain-wave analyzer is in normal operation and can measure changes in brain waves over time. They shows the energy change according to the frequency of EEG according to the change of the horizontal axis, which is the time flow. This is expressed as the band of the distribution component starting from 0 on the vertical axis. Table 1 shows the characteristics of the gamma, theta, alpha, and beta wave components. In addition, the improvement of the accuracy of the calculation is analyzed by superimposing $30 \%$ of the continuous calculation and the time span.

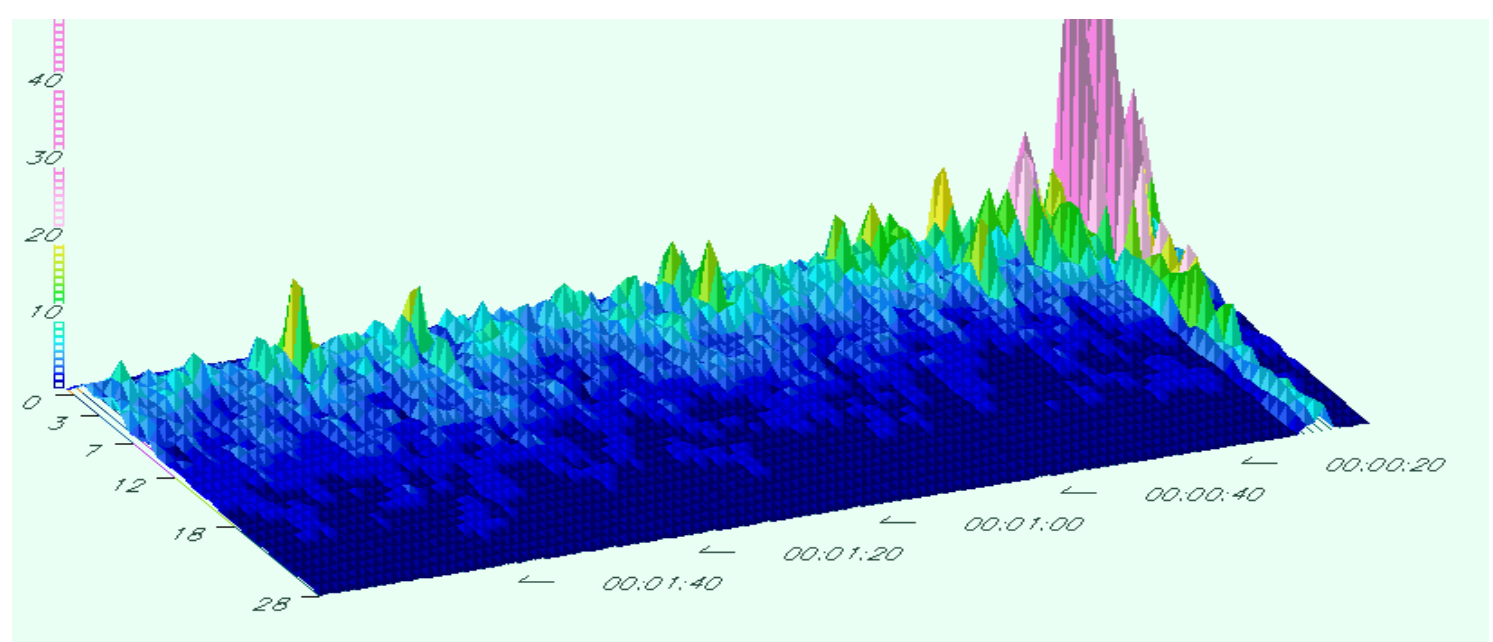

Fig. 11. Spectrogram analysis of synthesis sound 


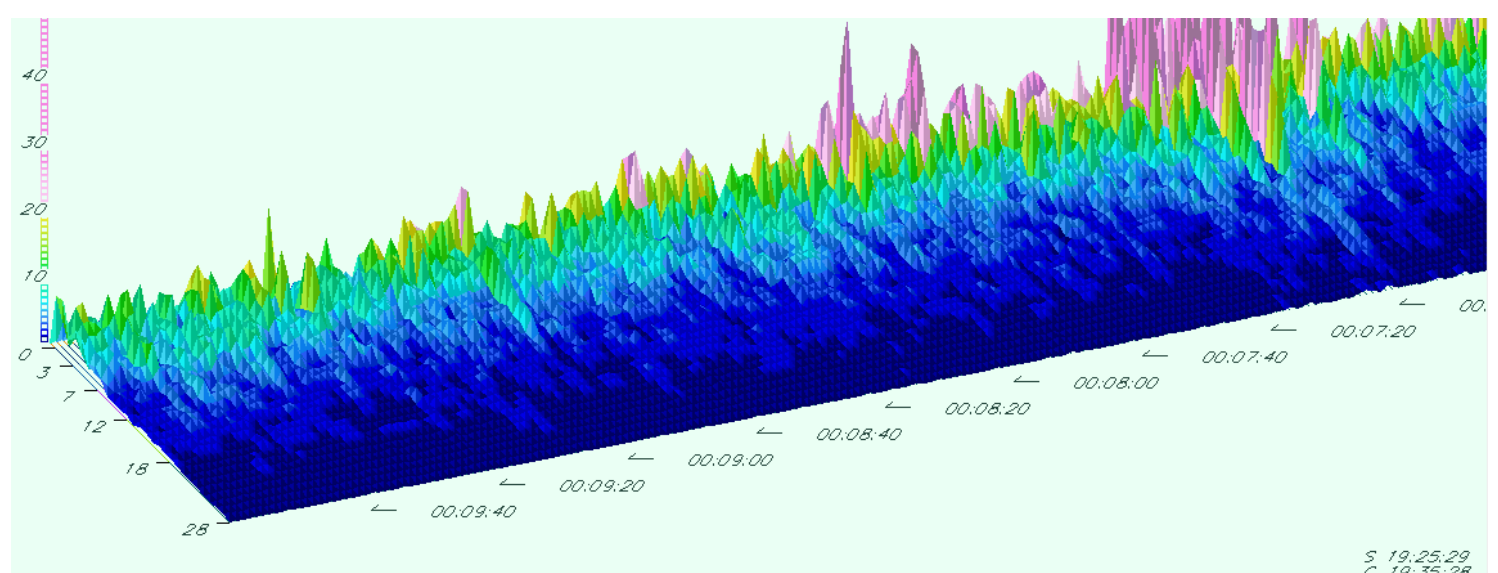

Fig. 12. Spectrogram analysis of synthesis sound

The EEG is a measure of the potential difference of the scalp according to the activity of the brain. The shape of the EEG may be different depending on the criteria at the time of measurement or the condition and situation of the experimenter. Thus, the main observation point is that the ratio of the energy occupied by each component of the EEG is judged based on the state of the brain at that time. In addition, the state of a person is judged by using a change in the ratio of the whole. In the initial state, at rest, the increase in beta waves can be seen as the brain being gradually awakened to an active state, and the increase in delta and theta waves gradually changes to the sleep or rest state. In Fig.s 11 and 12, such a change conspicuously does not appear.

\subsection{Experimental Results on Concentration by EEG Analysis}

The results of the EEG measurement were based on a spectrogram analysis and a circular graph showing the energy graph of the EEG component and the ratio of the average EEG of the selected intervals according to the time. In addition to the spectrogram, Fig. 13 shows the energy change of each component. It shows the energy distribution for each filtered component. This is the data of the beginning interval of the graph extracted from SW. The change of energy in the spectrogram of each EEG component is checked, and the composition ratio in the required interval is checked to determine whether the brain is awake or inactive. Fig. 13 shows a lot of delta and theta waves. There are a lot of EEGs that correspond to the slow-wave, so that the brain is resting, or not doing an activity. In addition, the blue and purple lines corresponding to the beta waves are distributed at a low level, and the stress is also low. 


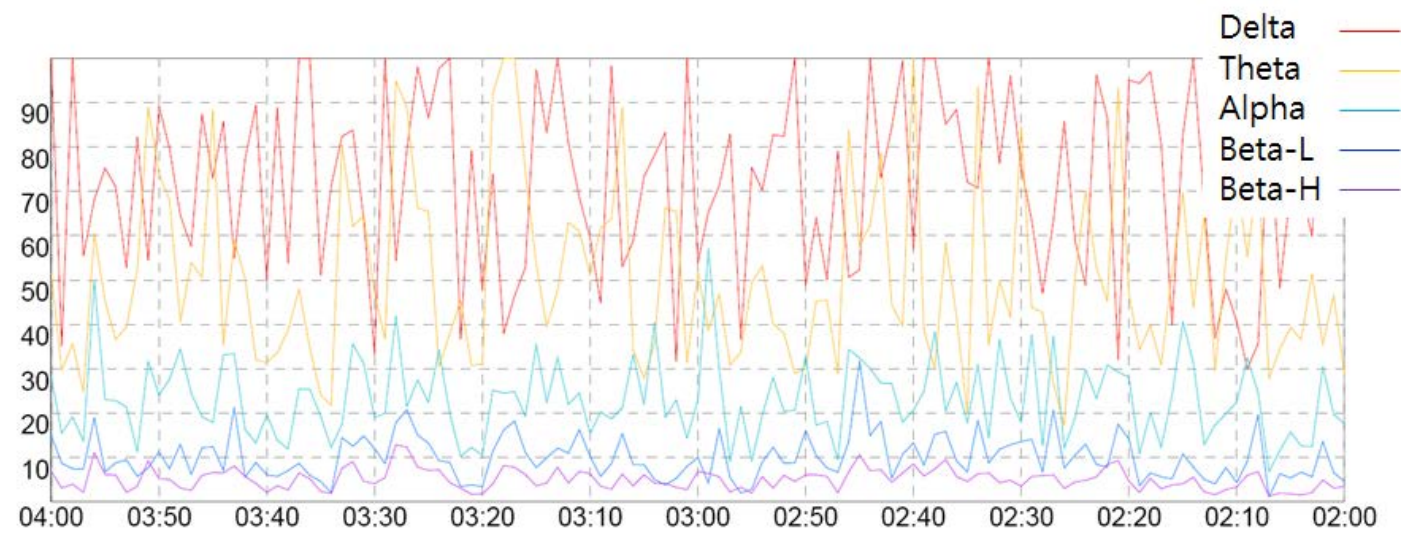

Fig. 13. Graph of variation of EEG components over time

Fig.s 14-19 show the average of the interval of the EEG analysis results. The resulting graph was saved and attached to the screen of the EEG analysis program, Neuro-Spec. The left side of the average EEG graph is the left brain, and the right side is the energy distribution of the right brain as a percentage. The left side of the figure shows the EEG distribution, while the right side shows the EEG distribution. The following figures show the interval averages of the EEG analysis results. The result graph was saved, and attached to the screen of the EEG analysis program. Fig. 14 shows the EEG at the beginning of measurement, as can be seen in Fig. 11, where the specificity of the EEG occupies about $60 \%$ of the EEG, such as delta and theta. The EEG was maintained for about two minutes after the experiment started; Beta waves increased in the middle; the results show that the subject awoke. A ratio of alpha of about $20 \%$ is mixed with appropriate stress near the start of the experiment. The state of the EEG that is the standard of the experiment established the status by measuring the rate of increase of the slow-wave, and the variation of the ratio of the beta wave to the alpha wave at the corresponding ratio.
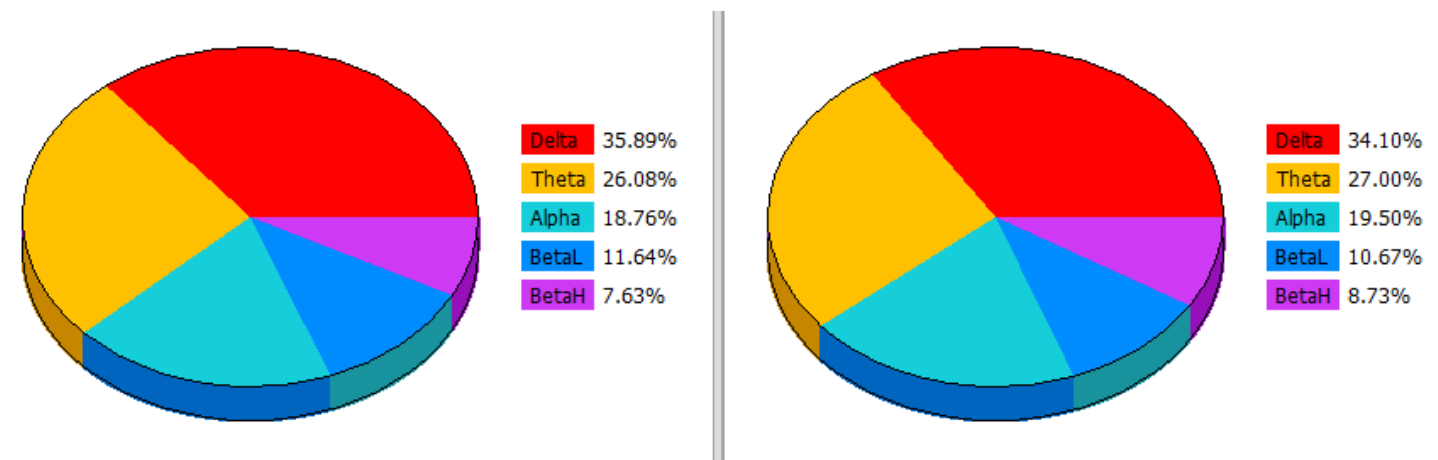

Fig. 14. Average EEG of start duration

Fig. 15 shows the average EEG after about 15 minutes of the experiment. Compared to Fig. 14, the ratio of slow-wave delta and theta increases by about $3 \%$. The ratio of alpha appears to have increased by $3 \%$. In addition, the beta wave is reduced by about $6 \%$, so the tension seems to have eased a little. After 15 minutes of experiment time, there was a slight change in the EEG, but it was judged that this was not a big change or a state of rest or 
concentration. As the slow-wave increases, the tension seems to be more relaxed than at the beginning of the experiment. However, it seems that the concentration has been maintained.
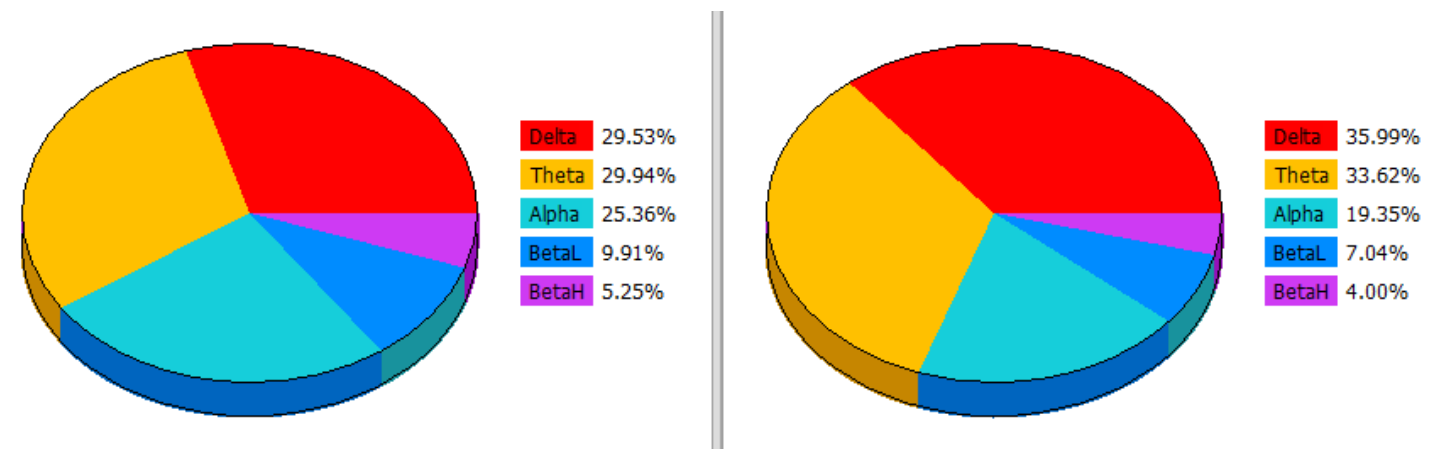

Fig. 15. Average EEG 15min later

Fig. 16 shows the average EEG after about 55 minutes of the experiment. Fig. 16 shows that although the concentration is lower than the state after 15 minutes in Fig. 15, the concentration is maintained. It can be concluded that the composition of the EEG changes as the rate of the alpha wave decreases by $3 \%$, the rate of the delta wave increases by $10 \%$ over the previous state, and the experiment continues. Nevertheless, only a small change in the ratio of beta is maintained. In comparison with the initial EEG of the experiment, it can be seen that the degree of concentration decreased as the slow wave increased and the fast wave decreased. However, the amount of beta waves is maintained, and we judged that it did not completely go to the drowsiness state.
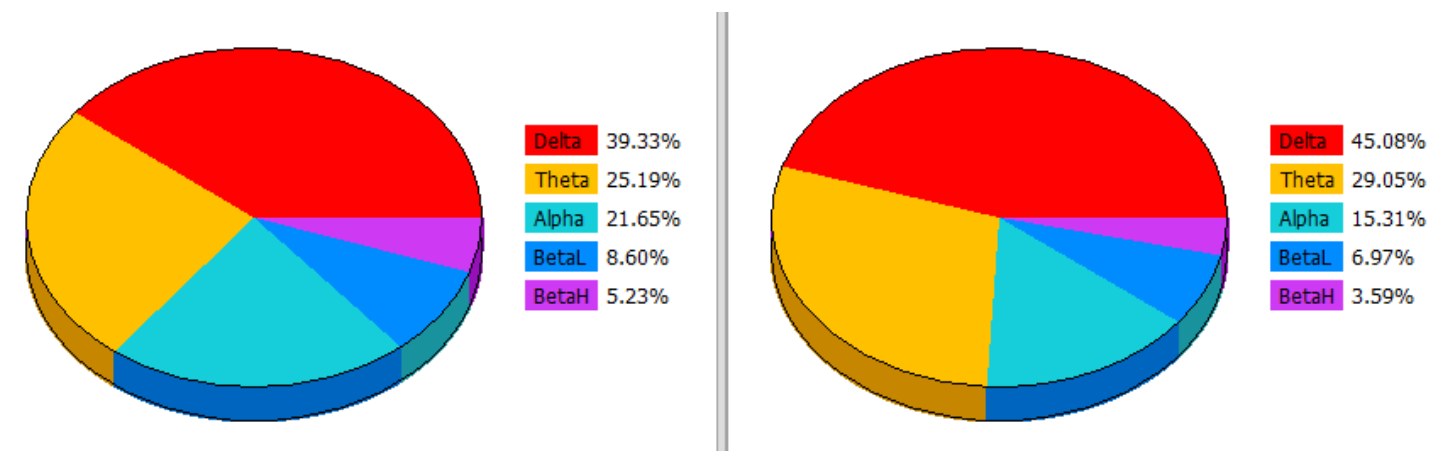

Fig. 16. Average EEG 55min later

The following experimental results show that there was no functional sound during the same period. Fig. 17 shows the average EEG after about 25 minutes of the experiment. Compared with Fig. 15, there are many differences. Rather, it is similar to the EEG when the concentration declined after 55 minutes in Fig. 16, and the activity of the brain is further reduced because the proportion of the delta wave is high in the slow wave. The ratio of the beta wave is also reduced, and we judged that it is not a quick transition or decision-making. Compared to Fig. 14, the fast-wave is reduced by $15 \%$, and the slow wave by $15 \%$. We judged that drowsiness was coming. 

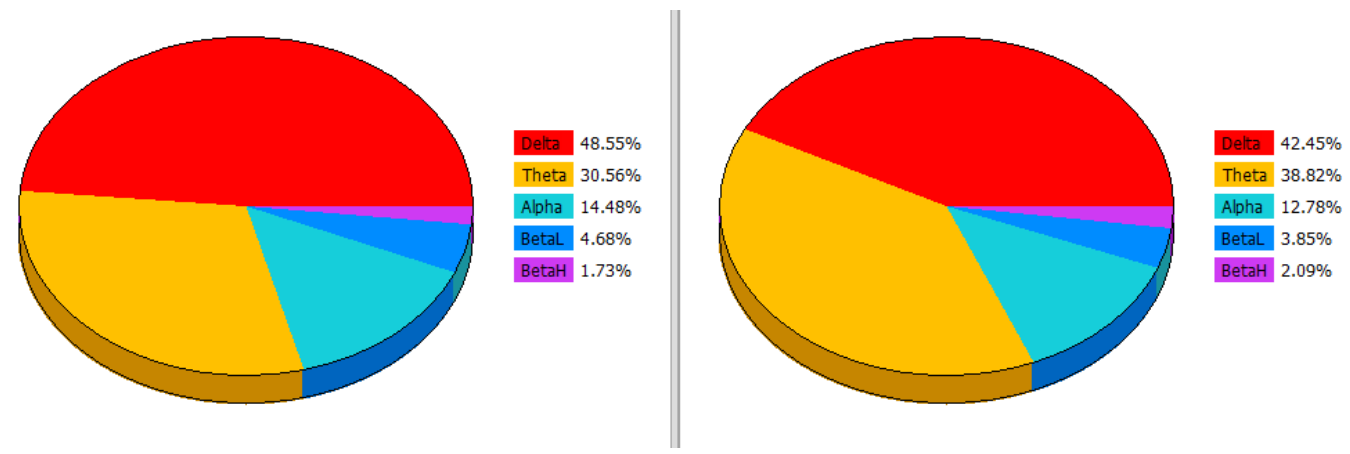

Fig. 17. Average EEG of $25 \mathrm{~min}$ later without functional sound

\subsection{Experimental results for short time resting through EEG analysis}

Fig.s 16 and 17 show the EEG when sound is used to induce slow EEG during rest. Fig. 16 shows the results after 10 minutes of hearing the source in arousal and concentration; Fig. 17 shows that the EEG is in a resting state. In the results of the analysis of the brain, particularly the right brain, the rate of the EEG is faster, and alpha is reduced to less than $25 \%$; delta indicating sleep occupies a large proportion.
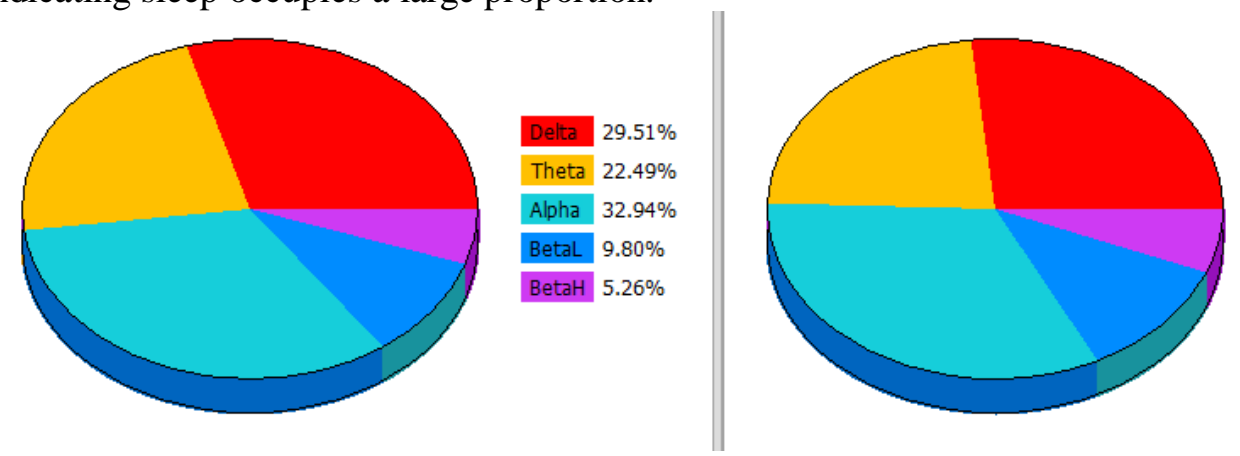

Delta $26.70 \%$ Theta $22.76 \%$ Alpha $32.97 \%$ BetaL $11.48 \%$ BetaH $6.10 \%$

Fig. 18. Average EEG of awake status
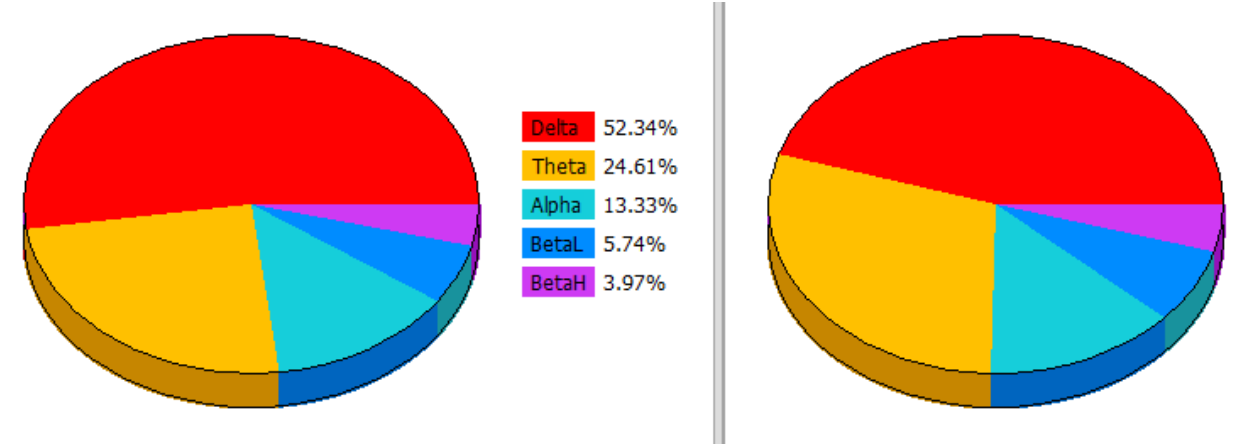

Delta $45.19 \%$

Theta $29.46 \%$

Alpha $13.65 \%$

BetaL $7.16 \%$

BetaH $4.54 \%$

Fig. 19. Average EEG 10min after sleep is induced 


\subsection{Discussion of experimental results}

In this study, we introduced technologies to prevent drowsy driving. These included a noise-reduction audio system, and a functional sound. The acoustic system showed a noise reduction of about $7 \mathrm{~dB}$. In addition, the functional sound can be played independently of the vehicle audio system. The system uses an anti-phase signal to reduce various noises associated with vehicle operation, and furthermore, generates drowsiness prevention. The signal-to-noise ratio indicates that the SNR is improved by about $10 \mathrm{~dB}$, compared to the case without the system. Because the system runs independently of the car audio system, the subject can listen to their favorite music or radio as on a regular car audio. The functional sound source was gradually supplied to the driver according to the vehicle's running and surrounding noise level. The sudden supply of sound sources tends to surprise drivers, or causes a sense of heterogeneity with the previous sound environment, so abrupt use is avoided.

In order to confirm the drowsiness prevention sound, a simulation environment similar to the drive was constructed, and the composition of the EEG with and without the sound was analyzed. In the course of time, we used a change in the composition of brain waves to maintain alertness, and to decide whether to keep concentration. When experimenting with functional sound sources, the concentration was slightly lowered, but we judged that drowsiness did not occur. However, we confirmed that when the sound source was not used, the EEG was composed of slow waves. It also responded to the sound that induces slow brain waves to increase efficiency during short breaks. Although it is necessary to perform additional tests and other verification procedures, it can be judged that based on the above experiments, the proposed sound is effective.

\section{Conclusion}

Advances in technology have made it possible to move people and goods by means of driving vehicles. However, depending on the physical and psychological health of the driver, a vehicle may cause accidents, including accidents that affect others. In particular, driving when suffering from insufficient sleep can lead to drowsiness, which could be more dangerous than drunk driving. It is important for a driver to relax in the middle of driving, and to relieve fatigue, in order to prevent accidents caused by such sleepy driving. In particular, drivers who use large trucks to transport logistical goods, or who transport passengers on buses, do not always follow the law. Trucks and buses can cause serious accidents, because they are large and heavy vehicles.

This research explores ways to influence the human brain and cognition using specific sounds. If human cognition and brain-wave response change when hearing a certain sound, human attitude and behavior can also change. Thus, a certain sound makes it possible for a person to concentrate more efficiently, or to relax efficiently. In the proposed method, a device using a bone-conduction speaker with an ANC function in the driver's seat and a drowsy-driving sound source are introduced. The device consists of a microphone for monitoring the noise in the headrest, a phasing device, and an amplifier bone-conduction speaker. The sound generated by this device reduces surrounding noise, and produces sounds to maintain concentration and prevent drowsy driving. This device suppresses the noise by about $7 \mathrm{~dB}$, and can also mask another (3-8) dB of ambient noise using a functional sound. Finally, the driver can suppress (10-15) dB of noise from the outside, and listen to sounds with increased concentration. We confirmed that the EEG result with the functional sound 
source maintained concentration and awakefulness. An EEG more than $10 \%$ faster than that without the functional sound source and awakening is also high. We also confirmed that a slow EEG leads to highly efficient rest, even during short breaks.

In this study, only the simulation was performed, because it is dangerous to measure the EEG during actual vehicle operation. In addition, in applying the functional sound source, the feedback and cognition of the user's preference and condition are not complete. In future work, we will carry out a study to add a sound source by using the sound of drowsy driving according to additional experiment and preference.

\section{References}

[1] Laverne C. Johnson, William L. MacLeod, "Sleep and awake behavior during gradual sleep reduction," Perceptual and Motor Skills, Vol. 36, No. 1, pp. 87-97,1973. Article (CrossRef Link)

[2] C. Bougard, M. C. Lepelley, and D. Davenne, "The influences of time-of-day and sleep deprivation on postural control," Experimental brain research, Vol. 209, No.1, pp.109-115, 2011.

[3] Jae Sik Lee, Bi A Kim and Wan Suk You, "The effects of alcohol and fatigue on divided attention task and driving performance using a driving simulator," The Korean Journal of Industrial and Organizational Psychology, vol. 12, no. 2, pp. 91-107, November, 1999. Article (CrossRef Link)

[4] H.J. Park "Functional device field of vehicle for safe driving - Patent Trend Analysis," Patent, Patent Information Promotion Center, vol. 21, no. 76, pp. 34-43, 2007.

[5] Dong Im Lee and Tae Gye Lee, "Study on the expansion of the scope of fatal driving style crime," Seoul Law Review, vol. 26, no. 3, pp.293-319, November, 2018. Article (CrossRef Link)

[6] Hwang Soo Chun, "A study on the ADAS drowsiness prevention technology," in Proc. of Symposium of the Korean Institute of Communications and Information Sciences, pp. 224-225 June, 2018. Article (CrossRef Link)

[7] Gi deok Lee, Jong soo Kum, Dong gyu Kim, Jung hwan Oh, Min soo Kim and Soo jin Yu, "A study on drowsiness prevention using blowing to face during driving," in Proc. of The Korean Society of Automotive Engineers, pp. 1880-1884, November, 2012. Article (CrossRef Link)

[8] Byung Chan Chang, Jung Eun Lim, Hae Jin Kim, and Bo Hyeok Seo, "A study of classification of the level of sleepiness for the drowsy driving prevention," SICE Annual Conference, September, 2007. Article (CrossRef Link)

[9] Hyung Woo Park, Seong Geon Bae and Myung Jin Bae, "A study on drinking judgment by the pitch analysis of speech signal," International Journal of Engineering and Technology, vol. 7, no. 6, pp. 2304-2308, 2016.

[10] Ki chun Jo, Jun soo Kim, Dong chul Kim, Chul hoon Jang and Myoung ho Sunwoo, "Development of autonomous car-part I distributed system architecture and development process," IEEE Transactions on Industrial Electronics, vol. 61, no. 12, pp. 7131-7140, 2014. Article (CrossRef Link)

[11] 'Temporary resarea installation and management instructions,' Ministry of Land, Infrastructure and Transport, Administrative Rule (Ordinance, Sample, Notification), 2017.

[12] Gideok Lee, Jongsoo Kum, Donggyu Kim, Junghwan Oh, Minsoo Kim, Soojin Yu, "A Study on Drowsiness Prevention using Blowing to Face during Driving," Proceedings of The Korean Society Of Automotive Engineers, 2012.11, pp.1880-1884. Article (CrossRef Link)

[13] Hyeon Ku Park, Yong Gyu Shin, Hang Kim, Min Jeong Song, Sun Woo Kim, "Evaluation of Sound Quality for Urban Environmental Sound," Proceedings of The Korean Society for Noise and Vibration Engineering, pp.529-534, 2005.

[14] W. Park, S. Lee, and S. Lee, Fundamentals of Sound Engineering. Seoul, Korea : Charsong, 2009.

[15] María Corsi-Cabrera, Enrique Pérez-Garci, Yolanda Del Río-Portilla, Enrique Ugalde, Miguel Angel Guevara, "EEG Bands During Wakefulness, Slow-Wave, and Paradoxical Sleep As a Result of Principal Component Analysis in the Rat," Sleep, Volume 24, Issue 4, PP. 374-380, 2001. Article (CrossRef Link) 
[16] S.W. Jee, H.W Park and M.J. Bae, "A Study on virtual car engine sound design according to sine wave harmonics frequency bandwidth," The Korean Institute of Communications and Information Sciences. Vol. 60, pp.1527-1528, 2016.

[17] Doo-Heon Kyon, Myung-Jin Bae, and Jeong-Hee Lee, “An analysis of the acoustic characteristics of forest sounds, " in Proc. of Meetings on Acoustics 167ASA, Vol. 21, No. 1, Acoustics Society America, 2014. Article (CrossRef Link)

[18] Daekwon Kim, "Criminal Psychological Considerations and Countermeasures against Cyber Drugs(i -Doser)," The Journal of Korean Crime Psychology Research, vol.6, no.1, pp. 3-29, 2010. Article (CrossRef Link)

[19] H.W. Park and S.W. Hahm, "Improvement of Concentration and Relaxation Efficiency through Specific Sounds: A convergent Study of Electroenc-ephalography and Cognitive Approach,” in Proc. of The 10th International Conference on Internet (ICONI 2018), Cambodia, 2018.

[20] Geun-Ho Lee, "Effects of Stress and Sleep Deprivation on Human Postural Control," THE KOREAN JOURNAL OF STRESS RESEARCH, Vol. 18, No. 4, 2010, pp.327-335.

[21] Y. Bereshpolova, C.R. Stoelzel, J. Zhuang, Y. Amitai, J.M. Alonso and H.A. Swadlow, "Getting drowsy? Alert/nonalert transitions and visual thalamocortical network dynamics," Journal of Neuroscience, Vol. 31, No. 48, 2011, pp.17480-17487.

[22] Dong wook Lee, Seung won Oh, Seong kook Heo and Min soo Hahn, "Drowsy driving detection based on the driver's head movement using infrared sensors," in Proc. of 2008 Second International Symposium on Universal Communication, IEEE, December, 2008. Article (CrossRef Link)

[23] Haruo Matsuo, Abdelaziz Khiat, "Prediction of drowsy driving by monitoring driver's behavior," in Proc. of the 21st International Conference on Pattern Recognition (ICPR2012), IEEE, November, 2012. Article (CrossRef Link)

[24] S Lim and D Lee, "Real-time eye tracking using ir stereo camera for indoor and outdoor environments," KSII Transactions on Internet and Information Systems, vol. 11, no. 8, 2017. Article (CrossRef Link)

[25] SF Ali and MT Hassan, "Feature based techniques for a driver's distraction detection using dupervised learning algorithms based on fixed monocular video camera," KSII Transactions on Internet \& Information Systems, vol. 12, no. 8, 2018. Article (CrossRef Link)

[26] Sandberg, David, and Mattias Wahde, "Particle swarm optimization of feedforward neural networks for the detection of drowsy driving," in Proc. of 2008 IEEE International Joint Conference on Neural Networks (IEEE World Congress on Computational Intelligence), IEEE, June, 2008. Article (CrossRef Link)

[27] Sakairi, Minoru, and Masahito Togami, "Use of water cluster detector for preventing drunk and drowsy driving," SENSORS, 2010 IEEE, IEEE, November, 2010. Article (CrossRef Link)

[28] Atsuo Murata, Taiga Koriyama and Takehito Hayami, "A basic study on the prevention of drowsy driving using the change of neck bending angle and the sitting pressure distribution," in Proc. of 2012 SICE Annual Conference (SICE), IEEE, August, 2012. Article (CrossRef Link)

[29] Lunbo Xu, Shunyang Li, Kaigui Bian, Tong Zhao and Wei Yan, "Sober-Drive A smartphoneassisted drowsy driving detection system," in Proc. of 2014 International Conference on Computing, Networking and Communications (ICNC), IEEE, February, 2014.

Article (CrossRef Link)

[30] Joel Gonçalves, Riender Happee and Klaus Bengler, "Drowsiness in conditional automation, proneness, diagnosis and driving performance effects," in Proc. of 2016 IEEE 19th International Conference on Intelligent Transportation Systems (ITSC), IEEE, November, 2016.

Article (CrossRef Link)

[31] Erika Abe, Koichi Fujiwara, Toshihiro Hiraoka, Toshitaka Yamakawa and Manabu Kano, "Development of drowsy driving accident prediction by heart rate variability analysis," in Proc. of Signal and Information Processing Association Annual Summit and Conference (APSIPA), 2014 Asia-Pacific, IEEE, December, 2014. Article (CrossRef Link) 
[32] Ying ying Jiao and Bao Liang Lu, "Detecting driver sleepiness from eeg alpha wave during daytime driving," in Proc. of 2017 IEEE International Conference on Bioinformatics and Biomedicine (BIBM), IEEE, November, 2017. Article (CrossRef Link)

[33] Arkhom Songkroh and Werasak Kurutach, "An intelligent hybrid approach for detection of drowsy driving risk in real environments," in Proc. of 2017 14th International Conference on Electrical Engineering Electronics, Computer, Telecommunications and Information Technology (ECTI-CON), IEE, June, 2017. Article (CrossRef Link)

[34] Hyung Woo Park and Sangmin Lee, "IoT Based Performance Measurement of Car Audio Systems in Korean Recreation Vehicles,” Journal of Internet Computing and Services(JICS), Vol. 18, No. 1, pp. 57-64, Feb., 2017. Article (CrossRef Link)

[35] H.W. Park, M.S. Kim, and M.J. Bae, "Improvement of voice quality and prevention of deafness by a bone-conduction device,” Biotechnology and Biotechnological Equipment, Taylor \& Francis, Vol. 14, No. 28, S14-S20, 2014. Article (CrossRef Link)

[36] Noppadon Jatupaiboon, Setha Pan-ngum, Pasin Israsena, "Emotion Classification using Minimal EEG Channels and Frequency Bands," in Proc. of 2013 10th International Joint Conference on Computer Science and Software Engineering (JCSSE), Maha Sarakham, 2013, pp. 21-24. Article (CrossRef Link)

[37] Eun-Jin Cheon, "Electroencephalographic Changes in Depression," J Korean Soc Biol Ther Psychiatry, Vol 19, No 2, pp. 93-100, 2013.

[38] Sangwoo Hahm,, “Attitudes and Performance of Workers Preparing for the Fourth Industrial Revolution,” KSII Transactions on Internet and Information Systems, vol. 12, no. 8, pp. 40384056, August, 2018.

[39] Sangwoo Hahm, "Information sharing and creativity in a virtual team: Roles of authentic leadership, sharing team climate and psychological empowerment," KSII Transactions on Internet and Information Systems, vol. 11, no. 8, pp. 4105-4119, August, 2017.

[40] L. R. Rabiner, and R. W. Schafer, Introduction to digital speech processing, Foundations and Trends® in Signal Processing, 2007.

[41] M. Fukumoto, “A finger-ring shaped wearable handset based on bone-conduction,” in Proc. of Ninth IEEE International Symposium on Wearable Computers (ISWC'05), October, 2005. Article (CrossRef Link)

[42] Irwansyah and Tsuyoshi Usagawa, “Application of active control technique on a bone conduction headphone for estimating a cross-talk compensation filter,” in Proc. of TENCON 2017 - 2017 IEEE Region 10 Conference, November, 2017. Article (CrossRef Link)

[43] H.W. Park, H.I. Koh and S.H. Jang, "NOISE REDUCING SYSTEM USING BONE CONDUCTION SPEAKER,” (KR)patent: 1014568870000, 2014. 


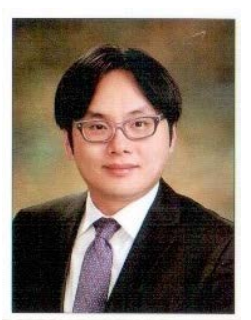

SangWoo Hahm is currently an assistant professor of College of Business Administration at Soongsil university, Seoul, Korea. He received his Ph.D, MS and BS in School of Business Administration from Soonsil University. His current research interests include e-business, management, The fourth industrial revolution, organizational behavior, interdisciplinary research and the like.

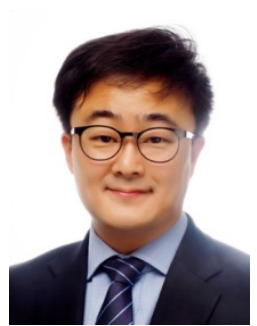

Hyung Woo Park is received a Ph.D., an M.S., and a B.S. in Electrical Engineering from Soongsil University. He is an assistant professor at the School of Information Technology, Soongsil University, Seoul, Korea. His current research interest includes Sound Signal Processing, Big Data Analysis, Voice Analysis, Noise Reduction System, Wave Field Aynthesis, and Internet of Things. 